\title{
Effect of the Corrected Ionization Potential on the HHG Transition Rate in a Linearly Polarized Laser
}

\author{
V. Petrović* , H. Delibašić and K. Isaković \\ Department of Physics, Faculty of Science, University of Kragujevac, Serbia \\ (Received April 26, 2018; in final form November 2, 2018)
}

\begin{abstract}
In this paper, we theoretically described the influence of the ponderomotive and the Stark shift on the highorder harmonic generation's transition rate for the cases of noble and alkali atoms in nonrelativistic, linearly polarized laser field. To describe harmonic generation, we used the analytical formula by Frolov et al. which is derived for a weakly bound electron in the tunneling limit and modified it in way to include mentioned effects. We showed that the inclusion of these effects affects the high-order harmonic generation's rate and that for the same conditions, the intensity of the alkali harmonics were considerably weaker compared to the intensity of noble harmonics. Also, the Stark shift for the alkali atoms induces not only decrease of the peak heights i.e. decrease of the ionization yield, but also the peak broadening. At the end, we analyzed the influence of the beam shape on the behavior of obtained theoretical curves. We considered two types of laser field shape, Gaussian and Lorentzian. It is shown that the high-order harmonic generation's rate depends on the spatial distribution of laser beam profiles.
\end{abstract}

DOI: 10.12693/APhysPolA.134.1170

PACS/topics: HHG rate, beam shape, effective ionization potential

\section{Introduction}

It is known that in the past, extensive studies have advanced our comprehension of the interaction of intense lasers with matter [1-3]. In order to provide a basis for understanding the physical picture of this kind of interaction, a wide range of experiments were performed [1, 2]. After extensive studies, it was shown that the laser-matter interaction in the high field regime is characterized by strongly non-linear effects. This kind of process led to discovery of high-order harmonic generation (HHG) $[2,4]$ and high-energy above-threshold ionization (HATI) [3]. Concretely, HHG spectroscopy has proved to be very promising for revealing various types of structural information about atoms and simple linear molecules [5-7] and it is the most convenient source of attosecond ( 1 as $\left.=10^{-18} \mathrm{~s}\right)$ pulses [8]. Because of that, HHG has been studied both experimentally and theoretically $[9-12]$.

Many features of HHG spectra can be explained by the semiclassical three-step model. In the first step the electron is released into continuum by tunnel ionization through the barrier formed by the Coulomb potential and the laser field. Its subsequent motion can be treated classically [13]. Tunnel ionization occurs when the Keldysh parameter [14], defined as a ratio of two energies, $\gamma=\sqrt{I_{p} / 2 U_{p}}$, is less than or much less than unity, $\gamma \ll 1$, where $I_{p}$ is unpertubed ionization potential and $U_{p}$ is the ponderomotive potential which represents the average kinetic energy of a free electron in a laser field, defined as $U_{p}=F^{2}\left(1-\varepsilon^{2}\right) / 4 \omega^{2}\left(1+\varepsilon^{2}\right)$,

\footnotetext{
* corresponding author; e-mail violeta.petrovickg@gmail.com
}

where $\varepsilon$ is ellipticity, $F$ is the field strength and $\omega$ is the laser field frequency [15]. For a linearly polarized laser field, $E(t)=E_{0} \cos (\omega t)$, ellipticity is $\varepsilon=0$, and $U_{p}$ becames $U_{p}=F^{2} / 4 \omega^{2}$. To simplify the notation, the atomic units are used, $e=m=\hbar=4 \pi \varepsilon_{0}=1$ throughout this paper [16]. After tunneling, an electron is accelerated by the oscillating laser field and later driven back to the target ion (second step) and third, it recombines with the ion to emit a high energy photon. The maximum photon energy produced by HHG, called the cutoff, is given by $I_{p}+3.17 U_{p}$ [17], i.e. the cutoff in the harmonic spectrum occurs at harmonics of order $N_{\max }=\left(I_{p}+3.17 U_{p}\right) / \omega$.

One of the semi-analytical quantum analyses of HHG process that confirm described three step model scenario is the formula developed by Frolov et al. [18]. In this paper we applied it on the atoms argon and potassium in order to analyze the influence of the strong field on the ionization potential and at the same time on harmonic rate.

\section{Theoretical concept}

As mentioned, in this paper we used and modified the simple analytic formula for harmonic rate, $W^{(l)}\left(E_{\Omega}\right)$, derived by Frolov [18], for the amplitudes and rates of harmonics generated by an electron bound in a short-range potential [19]:

$$
\begin{aligned}
& W^{(l)}\left(E_{\Omega}\right)=\frac{\left|I_{p}\right|^{2}}{E_{a t}} \frac{A_{n l}}{N}\left(\frac{\omega}{\left|I_{p}\right|}\right)^{4}\left(\frac{F_{0}}{\sqrt{\delta} F}\right)^{4 / 3} \frac{F_{0}}{\tilde{F}} \\
& \times \mathrm{e}^{-4 F_{0} / 3 \tilde{F}}\left(E / I_{p}-l\right)^{l+1} \mathrm{Ai}^{2}(\xi),
\end{aligned}
$$

where $E_{\Omega}$ is the harmonic energy, $A_{n l}$ is defined as $A_{n l}=\frac{\alpha^{3}}{\pi}\left(\frac{3}{4}\right)^{l} \frac{C_{n^{*} l}^{4}}{\kappa^{2} \tau_{c l}^{3}}, \quad \alpha=1 / 137$ is fine structure constant, $C_{n^{*} l^{*}}$ is the coefficient in the asymptotic form: $C_{n^{*} l}^{2}=\frac{2^{2 n^{*}}}{n^{*} \Gamma\left(n^{*}+l+1\right) \Gamma\left(n^{*}-l\right)}[20], n^{*}$ is the effective principal quantum number: $n^{*}=Z / \sqrt{2 I_{p}}, Z$ is the ion charge [13], $l$ is orbital angular momentum, $\kappa=\sqrt{2\left|I_{p}\right|}$, 
$\tau_{c l}=\tilde{\tau}^{(c l)}+\tilde{\tau}_{0}^{c l} \approx 4.086$ is the return time along a closed trajectory, $\tilde{F}=F\left|\cos \tilde{\tau}_{0}^{(c l)}\right|, \delta$ is dispersion parameter, $\mathrm{Ai}^{2}(\xi)$ is the Airy function where

$$
\begin{aligned}
& \xi=\frac{E-\varepsilon_{\max }}{\left|I_{p}\right|\left(\sqrt{\delta} F / F_{0}\right)^{2 / 3}}, \\
& \varepsilon_{\max }=\varepsilon_{c l}^{\max }+\Delta=2 \gamma^{-2} \sin ^{2}\left(\frac{\tau_{c l}}{2}\right)-\left.\left(\frac{\partial \tilde{\tau}_{0}}{\partial \tau}\right)\right|_{\tau=\tilde{\tau}(c l)}
\end{aligned}
$$

and

$$
F_{0}=\sqrt{2\left|I_{p}\right|^{3}}
$$

From Eq. (1) follows that the harmonic rate, $W^{(l)}\left(E_{\Omega}\right)$, among other, strongly depends on the ionization potential, $I_{p}$. On the other hand, when an atom is placed in a strong laser field the binding potential and the ionization process become increasingly influenced by the laser irradiation through the ponderomotive potential and the Stark shifts [21-23]. The ponderomotive potential is connected with the wiggle motion of charged particles in response to an applied laser field, while the Stark shift with the fact that atom's energy levels are altered in laser field [24]. It was experimentally observed that the energy levels are broadened and shifted due to laser-induced Stark effect [25]. This displacement of the energy level is determined by expression $I_{s t}=\frac{1}{2}\left(\alpha^{N}-\alpha^{1}\right) F^{2}$, where $\alpha^{N}$ is the static polarizability of the atom and $\alpha^{1}$ of its ion [26]. These two effects in the weak fields do not change substantially the ionization potential and can be neglected. But, in the strong field, Delone and Krainov [27] concluded that the Stark shift deepens the potential well (larger binding energy) and makes the atoms harder to ionize. This strongly affects the behaviour and shape of the harmonics and, because of that, in order to obtain better results with theoretical model we considered these effects with additional terms.

The Stark and the ponderomotive shifted ionization potential, $I_{p}^{\text {eff }}(F)$, can be written as

$$
\begin{aligned}
& I_{p}^{\mathrm{eff}}(F)=I_{p}(0)+I_{s t}+U_{p}= \\
& I_{p}(0)+\frac{1}{2}\left(\alpha^{N}-\alpha^{1}\right) F^{2}+\frac{F^{2}}{4 \omega^{2}} \frac{1-\varepsilon^{2}}{1+\varepsilon^{2}},
\end{aligned}
$$

where $I_{s t}$ is the shift caused by the Stark shift, and $U_{p}$ is ponderomotive potential. The influence of the inner electrons on the outer electron dynamics is included through the polarizability of the ion.

In order to analyze how the HHG rate is affected by effective ionization potential, $I_{p}^{\text {eff }}(F)$, we replaced unperturbed ionization potential, $I_{p}(0)$ with the effective, $I_{p}^{e f f}(F)$ in Eq. (1) and obtained the following expression:

$$
\begin{aligned}
& W_{\mathrm{eff}}^{(l)}\left(E_{\Omega}\right)=\frac{\left|I_{p}^{\mathrm{eff}}\right|}{E_{a t}} \frac{A_{n l}}{N}\left(\frac{\omega}{\left|I_{p}^{\mathrm{eff}}\right|}\right)^{4}\left(\frac{F_{0}}{\sqrt{\delta} F}\right)^{3} \frac{F_{0}}{F} \\
& \quad \times \exp \left(-\frac{4 F_{0}}{3 \tilde{F}}\right)\left(\frac{E}{I_{p}^{\text {eff }}-l}\right)^{l+1} A_{i}^{2}(\xi),
\end{aligned}
$$

i.e. in developed form with included both effects

$$
\begin{aligned}
& W_{\mathrm{eff}}^{(l)}\left(E_{\Omega}\right)=\frac{\left|I_{p}(0)+\frac{1}{2}\left(\alpha^{N}-\alpha^{1}\right) F^{2}+\frac{F^{2}}{4 \omega^{2}} \frac{1-\varepsilon^{2}}{1+\varepsilon^{2}}\right|^{2} \frac{\alpha^{3}}{\pi}\left(\frac{3}{4}\right)^{l} \frac{\left(\frac{2^{2 n^{*}}}{n^{*} \Gamma\left(n^{*}+l+1\right) \Gamma\left(n^{*}-l\right)}\right)^{2}}{\kappa^{2} \tau_{c l}^{3}}}{E_{a t}} \\
& \times\left(\frac{\omega}{\left|I_{p}(0)+\frac{1}{2}\left(\alpha^{N}-\alpha^{1}\right) F^{2}+\frac{F^{2}}{4 \omega^{2}} \frac{1-\varepsilon^{2}}{1+\varepsilon^{2}}\right|}\right)^{4}\left(\frac{\sqrt{2\left|I_{p}(0)+\frac{1}{2}\left(\alpha^{N}-\alpha^{1}\right) F^{2}+\frac{F^{2}}{4 \omega^{2}} \frac{1-\varepsilon^{2}}{1+\varepsilon^{2}}\right|^{3}}}{\sqrt{\delta} F}\right)^{4 / 3} \\
& \times \frac{\sqrt{2\left|I_{p}(0)+\frac{1}{2}\left(\alpha^{N}-\alpha^{1}\right) F^{2}+\frac{F^{2}}{4 \omega^{2}} \frac{1-\varepsilon^{2}}{1+\varepsilon^{2}}\right|^{3}}}{\tilde{F}} \exp \left(\frac{-4 \sqrt{2\left|I_{p}(0)+\frac{1}{2}\left(\alpha^{N}-\alpha^{1}\right) F^{2}+\frac{F^{2}}{4 \omega^{2}} \frac{1-\varepsilon^{2}}{1+\varepsilon^{2}}\right|^{3}}}{3 \tilde{F}}\right) \\
& \times\left(\frac{E}{I_{p}(0)+\frac{1}{2}\left(\alpha^{N}-\alpha^{1}\right) F^{2}+\frac{F^{2}}{4 \omega^{2}} \frac{1-\varepsilon^{2}}{1+\varepsilon^{2}}}-l\right)^{l+1} \mathrm{Ai}^{2}(\xi) .
\end{aligned}
$$

In Eq. (4) the general beam shape is assumed. Next, we discussed a different beam shape. We started with the Gaussian shaped laser pulse which is the simplest and often the most desirable type of beam provided by a laser source which allows the highest concentration of light. Gaussian beams can be represented in the following form [28]:

$$
F(\rho)=F \exp \left(-2\left(\frac{\rho}{R}\right)\right)
$$
where $\rho$ is the axial coordinate that is normal to the light ray [32] $\rho=R \sqrt{1+\left(\frac{\lambda \eta}{\pi R^{2}}\right)}, R$ is the radius of the laser beam and $F$ is the amplitude of the laser field strength. This is a simplified formula based on the presumption 
that the beam comes to its narrowest width (called the beam waist) at $z=0$.

In order to examine how pulse shape affects the HHG rate, we substituted Eq. (5) in Eq. (3) and obtained

$$
\begin{aligned}
& W_{2}^{(l)}\left(E_{\Omega}\right)=\frac{\left|I_{p}^{\mathrm{eff}, * *}\right|^{2}}{E_{a t}} \frac{A_{n l}}{N}\left(\frac{\omega}{\left|I_{p}^{\mathrm{eff}, * *}\right|}\right)^{4} \\
\times & \left(\frac{F_{0}}{\sqrt{\delta} F \exp \left(-2\left(\frac{\rho}{R}\right)^{2}\right)}\right)^{4 / 3} \frac{F_{0}}{F \exp \left(-2\left(\frac{\rho}{R}\right)^{2}\right)\left|\cos \tilde{\tau}_{0}^{(c l)}\right|} \\
\times & \mathrm{e}^{-4 F_{0} / 3 \tilde{F}}\left(E / I_{p}^{\mathrm{eff}, * *}-l\right)^{l+1} \mathrm{Ai}^{2}(\xi) .
\end{aligned}
$$

Although the Gaussian distribution is frequently assumed, there are a few other shapes, such as Lorentzian which can be represented as

$$
F(\rho)=F /\left(1+\left(\frac{\rho}{R}\right)^{2}\right) .
$$

Substituting Eq. (7) in Eq. (3), it can be check how this type of beam shape affects the HHG rate.

\section{Discussion}

We investigated the HHG rate induced by a linearly polarized laser field whose intensity varied between $10^{13} \mathrm{~W} \mathrm{~cm}^{-2}$ and $10^{16} \mathrm{~W} \mathrm{~cm}^{-2}$, for single ionized, $Z=1$, argon (Ar) and potassium atom $(\mathrm{K})$. We considered the tunneling regime, $\gamma=0.5<1$ and laser pulse with the wavelength $\lambda=800 \mathrm{~nm}$. We assumed that the electron velocity is small compared to the speed of light.

We started from the HHG curves obtained by Eq. (3), for harmonic order $12 \leq N \leq 14$. Figure 1 shows $3 \mathrm{D}$ graph for the argon atom rate (without (a) and with correction of ionization potential (b)), as well as the comparative review (2D graph) of both curves, (c).

We can see in Fig. 1a,b that both curves have almost the same behavior, but the rate with included effects is smaller than for the rate with unperturbed potential. With inclusion of effects, we see that the yield falls below for all intensities, which can be seen in 2D graph, Fig. 1c. Our theoretical analysis shows that $W^{(l)}\left(E_{\Omega}\right)$ is very sensitive on the Keldysh parameter, as well as the field intensity. The minimal change of those parameters strongly affects the harmonic rate. The shape of the harmonics is in accordance to [30].

Figure 2 shows $2 \mathrm{D}$ graph for HHG rate, $W_{\mathrm{eff}}^{(l)}\left(E_{\Omega}\right)$, as a function of the harmonic order. For both curves it is characteristic that the inclusion of the ponderomotive and the Stark shift induces the decrease of peak order i.e. changes the cut off energy (harmonic order). It was experimentally observed that the laser-induced Stark effect results in the peak shift and, at the same time, peak broadening. This leads to the decrease of the peak heights and decrease of the ionization yield [25]. This is expectable because additional energy is necessary for ionization in order to overcome higher level of the ionization potential. Figure 2a shows that in the case of argon atom
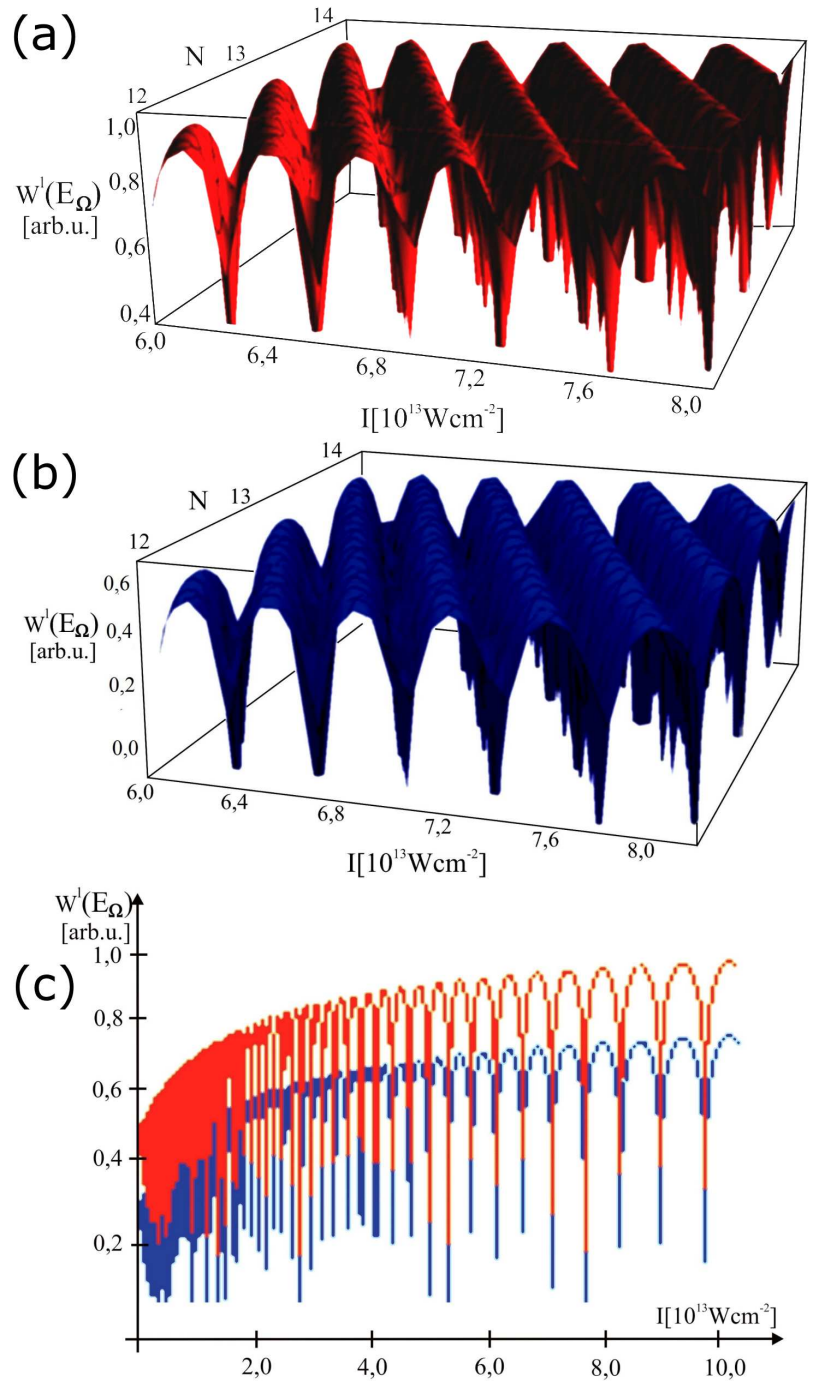

Fig. 1. 3D graph as a function of the field intensity, $F$, and harmonic order, $N$, for Ar atom (a) $W^{(l)}\left(E_{\Omega}\right)$ without any corrections of the ionization potential, (b) $W_{\text {eff }}^{(l)}\left(E_{\Omega}\right)$ with included effective ionization potential, (c) $2 \mathrm{D}$ comparative review of $W^{(l)}\left(E_{\Omega}\right)$ and $W_{\text {eff }}^{(l)}\left(E_{\Omega}\right)$. Field intensity varies within the range $F=8 \times 10^{13}-1 \times$ $10^{15} \mathrm{~W} \mathrm{~cm}^{-2}$ and the harmonic order $N=12,13,14$.

curves have almost the same shape and cut-off. The obtained cut-off for the harmonic order in Fig. $2 \mathrm{a}$ is in accordance with [31] for the same value of laser intensity. For potassium atom in Fig. $2 \mathrm{~b}$ there is a significant deviation in harmonic cut-off. The shape of the harmonics and achieved cut-off for the harmonic order for potassium are in accordance to [32]. As well, for potassium atom harmonics are slightly narrower compared to these of argon atom. It can be seen from Fig. $2 \mathrm{~b}$ that the influence of additional effects is larger for potassium atom [33].

In order to analyze in more detail the influence of observed effects on HHG rate, we included them sequentially (step by step) in Fig. 3. As a result, we obtained the following graphs. 

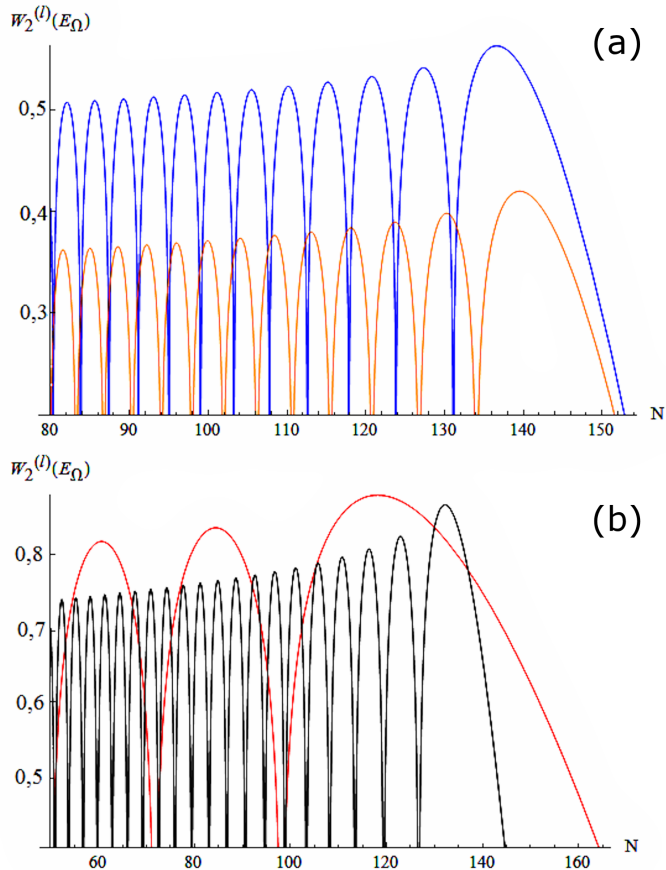

Fig. 2. 2D graph for $W_{\text {eff }}^{(l)}\left(E_{\Omega}\right)$ as a function of harmonic order $N$ for (a) Ar atom, (b) K atom. Field intensity is fixed on $F=8 \times 10^{14} \mathrm{~W} \mathrm{~cm}^{-2}$.

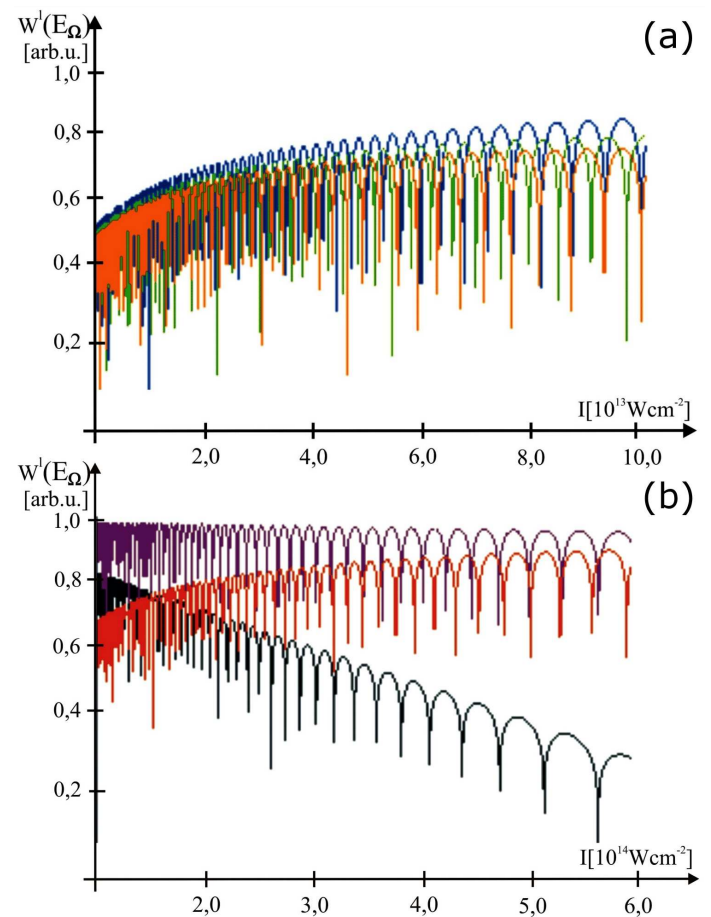

Fig. 3. Comparative review: (a) Ar atom, blue line HHG rate without any corrections of the ionization potential, green with ponderomotive and orange with ponderomotive and Stark shift, (b) K atom, red line without any correction, purple with included the ponderomotive and black line with ponderomotive and the Stark shift. Intensity varies within the range: $F=8 \times 10^{13}$ $6 \times 10^{14} \mathrm{~W} \mathrm{~cm}^{-2}$. For all theoretical curves the Keldysh parameter is $\gamma=0.5$.
A closer inspection shows faster decrease of HHG with included effect, $W_{\mathrm{eff}}^{(l)}\left(E_{\Omega}\right)$, with increasing field in comparison with $W^{(l)}\left(E_{\Omega}\right)$. The curve with both effects included is shifted vertically downward (orange line in 2D graph, Fig. 3a). Also there is a slight shift of the harmonic toward the lower field intensity when the ponderomotive potential is included (green solid line). As the intensity of the laser field is increased from $I=$ $9.7 \times 10^{13} \mathrm{~W} \mathrm{~cm}^{-2}$ there is the significant deviation of the HHG rate's curves.

From Fig. 3b black line, line with ponderomotive and the Stark shift, it can be seen the distinctive peak broadening as well [34]. The physical reason for this behavior of HHG rate for potassium atom is the Stark shift affecting the ground state. As Delone and Krainov predicted in [33] the influence of the Stark shift is larger in the case of alkali atoms and as a consequence the rate level for potassium atom is lower compared to argon atom. Figure 3 clearly shows that, i.e. significantly different behavior of potassium's curve. Now, it can be noted that the ground state shift caused by the ponderomotive and the Stark shift cannot be neglected in the analysis.

Next, we considered the influence of the spatial laser beam shape on the HHG rate. Lasers usually emit beams with a Gaussian profile, so we employ edit in our analysis.
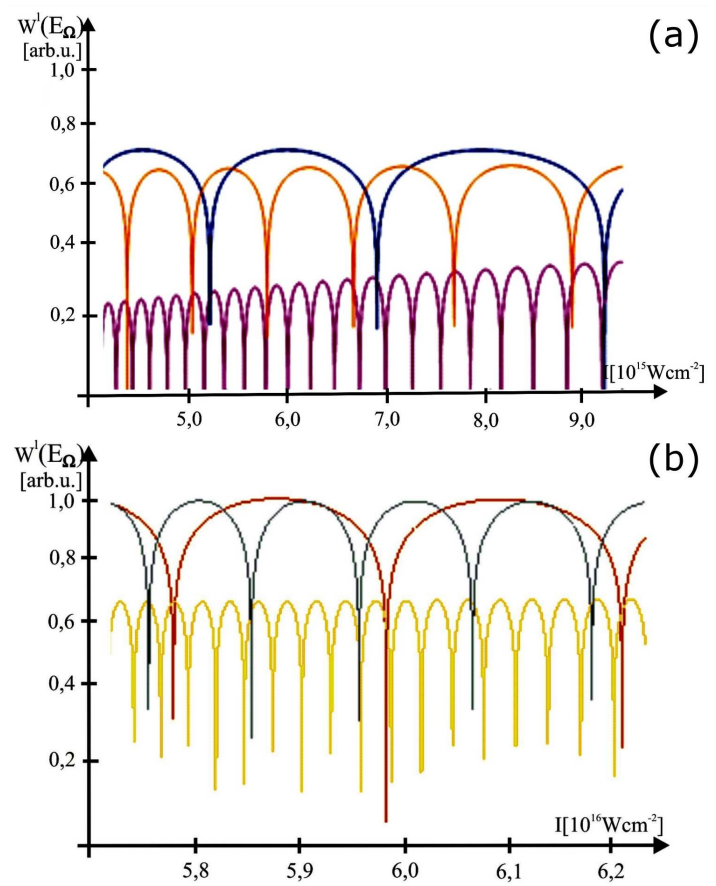

Fig. 4. Comparative review: (a) Ar atom, blue line HHG rate without any corrections of the ionization potential, magenta with spatial Gaussian pulse shape and orange with spatial Lorentzian pulse shape. Intensity varies within the range: $F=5 \times 10^{15}-9 \times 10^{15} \mathrm{~W} \mathrm{~cm}^{-2}$, (b) K atom, red line without any correction, yellow with with spatial Gaussian pulse shape and gray line with with spatial Lorentzian pulse shape. Intensity varies within the range: $F=5.6 \times 10^{14}-6.6 \times 10^{14} \mathrm{~W} \mathrm{~cm}^{-2}$. For all theoretical curves the Keldysh parameter is $\gamma=0.5$. 
For the presentation of the HHG rates a spatial Gaussian and Lorentzian pulse shape were used with intensities ranging from about $F=10^{14}-10^{15} \mathrm{~W} \mathrm{~cm}^{-2}$. In order to visualize this, we gave graphs for noble and alkali atoms, for the same conditions, Fig. 4.

Figure 4 shows an example of comparison between the generally assumed and specified laser beam shapes. It is obvious that there is a deviation between these two graphs, as well as between graphs for each atom particularly. For the argon atom the form of the spectrum changes dramatically with employed Gaussian beam profile.

The following graphs, Fig. 5, show 2D comparative review of the general, Fig. 5a, and the Gaussian beam shape, Fig. $5 \mathrm{~b}$, for HHG rate, $W_{\mathrm{eff}}^{(l)}\left(E_{\Omega}\right)$, as a function of the harmonic order.

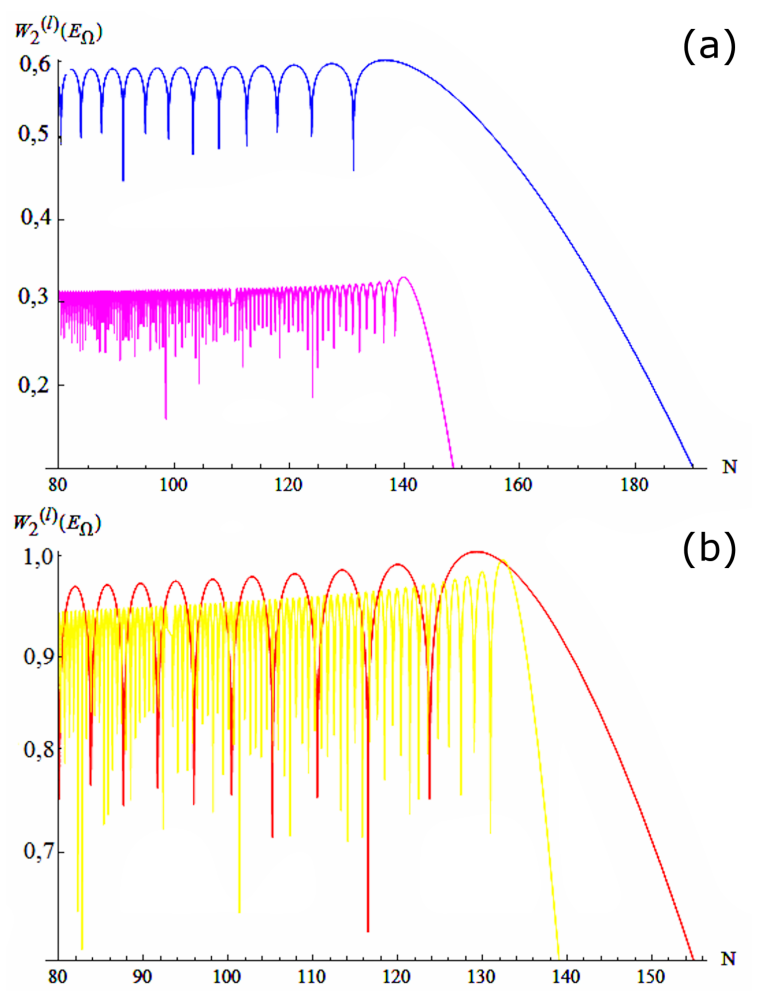

Fig. 5. Comparative review of the general and the Gaussian beam shape with included both effects in the ionization potential, and field intensity is $F=$ $8 \times 10^{14} \mathrm{~W} \mathrm{~cm}^{-2}$ : (a) Ar atom, the general (blue solid line) and the Gaussian beam shape (magenta solid line), (b) K atom, the general (red solid line) and the Gaussian beam shape (yellow solid line).

From Fig. 5 it can be seen that the inclusion of the Gaussian beam shape induces the decrease of the HHG rate $W_{\mathrm{eff}}^{(l)}\left(E_{\Omega}\right)$ and peak order i.e. changes the cut off energy (harmonic order). Also, Fig. 5a clearly shows that the curve with the Gaussian beam shape is shifted vertically downward. From Fig. 5b it is obvious that the discrepancy between the curves becomes more clear for higher harmonic order.
In order to shed more light on this deviation, we repeated the same procedure with the Lorentzian laser beam shape. As a result we obtained similar behavior of the observed curves.

\section{Conclusion}

We have theoretically investigated HHG rate for single ionization argon and potassium atom as a function of field intensity. We restricted ourselves to the case of nonrelastivic case of linearly polarized laser pulses. In addition, we discussed the influence of the spatial beam profile on HHG rate. We compared the obtained results with experimental data and obtain a very good agreement between theory and experiment.

\section{Acknowledgments}

The authors are grateful to the Serbian Ministry of Education, Science and Technological Development for financial support through Project 171020.

\section{References}

[1] P. Agostini, F. Fabre, G. Mainfray, G. Petite, N.K. Rahman, Phys. Rev. Lett. 42, 1127 (1979).

[2] S. Augst, D. Strickland, D.D. Meyerhofer, S.L. Chin, H. Eberly, Phys. Rev. Lett. 63, 2212 (1989).

[3] K.J. Schafer, Baorui Yang, L.I. DiMauro, K.C. Kulanderc, Phys. Rev. Lett. 70, 1599 (1993).

[4] M. Ferray, A. L'Huillier, X.F. Li, L.A. Lompre, G. Mainfray, C. Manus, J. Phys. B At. Mol. Opt. Phys. 21, L31 (1988).

[5] C. Vozzi, M. Negro, F. Calegari, G. Sansone, M. Nisoli, S. De Silvestri, S. Stagira, Nature Phys. 7, 823 (2011).

[6] S. Haessler, J. Caillat, W. Boutu, C. GiovanettiTeixeira, T. Ruchon, T. Auguste, Z. Diveki, P. Breger, A. Maquet, B. Carré, R. Taïeb, P. Salières, Nature Phys. 6, 200 (2010).

[7] O. Smirnova, Y. Mairesse, S. Patchkovskii, N. Dudovich, D. Villeneuve, P. Corkumn, M.Y. Ivanov, $\mathrm{Na}$ ture 460, 972 (2009).

[8] F. Krausz, M. Ivanov, Rev. Mod. Phys. 81, 163 (2009).

[9] P.M. Abanador, F. Mauger, K. Lopata, M.B. Gaarde, K.J. Schafer, J. Phys. At. Mol. Opt. Phys. 50, 035601 (2017).

[10] J.C.P. Koliyadu, S. Künzel, T. Wodzinski, B. Keitel, J. Duarte, G.O. Williams, C.P. João, H. Pires, V. Hariton, M. Galletti, N. Gomes, G. Figueira, J.M. Dias, N. Lopes, P. Zeitoun, E. Plönjes, M. Fajardo, Photonics 4, 25 (2017).

[11] H. Yun, S.J. Yun, G.H. Lee, C.H. Nam, J. Phys. B At. Mol. Opt. 50, 022001 (2017).

[12] A.A. Minina, M.V. Frolov, A.N. Zheltukhin, N.V. Vvedenskii, Quant. Electron. 47, 216 (2017).

[13] M.V. Ammosov, N. Delone, V.P. Krainov, Sov. Phys. JETP 64, 1191 (1986). 
[14] L.V. Keldysh, Sov. Phys. JETP 20, 1307 (1965).

[15] P. Antoine, A. L'Huillier, M. Lewenstein, P. Salières, B. Carré, Phys. Rev. A 53, 1725 (1996).

[16] R. McWeeny, Nature 243, 196 (1973).

[17] P.B. Corkum, Phys. Rev. Lett. 71, 1994 (1993).

[18] M.V. Frolov, N.L. Manakov, T.S. Sarantseva, A.F. Starace, J. Phys. B At. Mol. Opt. Phys. 42, 035601 (2009).

[19] M.V. Frolov, N.L. Manakov, A.F. Starace, Phys. Rev. A 78, 063418 (2008).

[20] B. Shan, Z. Chang, Phys. Rev. A 65, 011804 (2001).

[21] S.H. Autler, C.H. Townes, Phys. Rev. 100, 703 (1955).

[22] E.A. Volkova, A.M. Popov, O.V. Tikhonova, JETP 113, 394 (2011).

[23] M. Protopapas, C.H. Keitel, P.L. Knight, Rep. Progr. Phys. 60, 389 (1997).

[24] N.I. Shvetsov-Shilovski, M. Lein, L.B. Madsen, E. Räsänen, C. Lemell, J. Burgdörfer, D.G. Arbó, K. Tökési, Phys. Rev. A 94, 013415 (2016).

[25] K.L. Ishikawa, in: Advances in Solid State Lasers Development and Applications, Ed. M. Grishin, 2010, Ch. 19.
[26] P. Schwerdtfeger, Table of experimental and calculated static dipole polarizabilities for the electronic ground states of the neutral elements (in atomic units), 2017, accessed 25 September 2017.

[27] N.B. Delone, V.P. Krainov, Phys. Usp. 42, 669 (1999).

[28] I.I. Bondar, V.V. Suran, D.I. Bondar, Phys. Rev. A 88, 023407 (2013).

[29] C.G. Gomez, M.Sc. Thesis, Brno University of Technology, Brno, Czech Republic 2012, .

[30] X. Bian, A.D. Bandrauk, Appl. Sci. 3, 267 (2013).

[31] K.H. Hong, C.J. Lai, V.M. Gkortsas, S.W. Huang, J. Moses, E. Granados, S. Bhardwaj, F.X. Kärtner, Phys. Rev. A 86, 043412 (2012).

[32] C.T. Whelan, Fragmentation Processes: Topics in Atomic and Molecular Physics, Cambridge University Press, 2013, p. 98.

[33] N.B. Delone, V.P. Krainov, Phys. Usp. 41, 469 (1998).

[34] I.A. Burenkov, A.M. Popov, O.V. Tikhonova, E.A. Volkova, Laser Phys. Lett. 7, 409 (2010). 